\title{
Análisis de la capacidad de autocuidados en pacientes en diálisis peritoneal
}

\author{
Ana Julia Carrillo Algarra \\ Profesora titular facultad de enfermería, Magister en administración en salud Fundación Universitaria de Ciencias de \\ la Salud, Hospital de San José Carrera. Bogotá. Colombia
}

\section{Resumen}

Introducción: La Insuficiencia renal crónica es considerada una enfermedad de alto costo por sus efectos sobre la calidad de vida del paciente, la familia y el impacto en los servicios de salud, aspectos posibles de mitigar fortaleciendo el autocuidado. Objetivo: Medir la operatividad de la capacidad de autocuidado y establecer su relación con características sociodemográficas y clínicas en pacientes del programa de diálisis peritoneal en unidades renales de Bogotá, Colombia. Métodos: Estudio cuantitativo descriptivo transversal, población: 915 pacientes mayores de 18 años en diálisis peritoneal; se realizó muestreo probabilístico estratificado, se seleccionaron 105 pacientes en diálisis peritoneal automatizada y 174 en manual. Enfermeras especializadas en nefrología y urología les aplicaron tres instrumentos: Escala de Actividades de Autocuidado (ASA-A), Test de Morinski Green y formulario para caracterización sociodemográfica y clínica. Los datos se trataron mediante análisis descriptivo, utilizando tablas de frecuencia y correspondencias múltiples. Resultados: El promedio de la capacidad de autocuidado es de 78.7, desviación estándar 16.5 , el $72 \%$ de los pacientes obtuvo puntuaciones superiores al mismo. El $77.42 \%$ de los participantes se clasificó en el rango de operatividad de la capacidad de agencia de autocuidado alta, el $9.32 \%$ media y el $13.98 \%$ baja. La mediana se ubicó en 78 puntos, rango intercuartílico de 77-88, y la moda fue de 86 puntos. La puntuación máxima fue de 96 reportado por tres pacientes y la mínima fue 27 . En conclusión el $22.30 \%$ de los participantes requiere que el personal de salud diseñe estrategias que fortalezcan la operatividad de agencia de autocuidado.

Correspondencia:

Ana Julia Carrillo Algarra

Fundación universitaria de ciencias de la salud

Hospital de San José Carrera 19 N 8A-32

Bogotá D.C. Colombia

E-mail: ajcarrilloster@gmail.com
PALABRAS CLAVE

- AUTOCUIDADO

- DIÁLISIS PERITONEAL

- INSUFICIENCIA RENAL CRÓNICA

- ATENCIÓN DE ENFERMERÍA Y CUMPLIMIENTO DE LA MEDICACIÓN

The operability of the self-care agency capacity of patients in peritoneal dialysis

\section{Abstract}

Introduction: Chronic kidney disease is considered a high-cost disease due to its effects on the patient's life, including his family and the impacts it has on the facility providing healthcare. These problems can be mitigated with better self-care. Objective: To measure the operability of self-care capability and establish its relationship with socio-demographic and clinical characteristics for patients in the peritoneal dialysis program in nephrology units in Bogotá, Colombia. Methodology: A cross-sectional descriptive and quantitative study was done with a population of 915 patients of over 18 years of age in peritoneal dialysis. A stratified, probability sampling method was chosen and 105 patients in automatic peritoneal dialysis were selected, while 174 patients in manual peritoneal dialysis participated. Nurses specialized in nephrology and urology asked them to fill out three instruments: a self-care activities scale (ASA-A), a Morisky Green test and $a$ form to identify socio-demographic and clinical characteristics. Data were analyzed descriptively, using frequency tables and multiple correspondences. Results: The average score for self-care capability was 78.7 , with a standard deviation of $16.5 .72 \%$ of patients got a score above the average. $77.42 \%$ of patients were classified in the high operability range of self-care agency, $9.32 \%$ in the medium range and $13.98 \%$ in the low range. The median was 78 points with an inter-quartile range of 77-88 y and the mode was 86 points. Three subjects got the maximum score 
of 96 and the lowest was 27 . In conclusion, $22.30 \%$ of subjects require that the healthcare personnel design strategies to improve the operability of self-care agency.

\section{KEYWORDS}

- SELF-CARE

- PERITONEAL ANALYSIS

- CHRONIC KIDNEY DISEASE

- INFIRMARY ATTENTION, MEDICATION CONTROL

\section{Introducción}

"El autocuidado se refiere a las acciones basadas en prácticas derivadas de la cultura o la ciencia que son realizadas con total libertad por los individuos. Estas prácticas están dirigidas al individuo mismo o a condiciones u objetos en su ambiente. Se ejecutan en pos de la vida de la misma persona, su salud o bienestar"l 1 .

Según Flores (2009)², la insuficiencia renal crónica (IRC) es un problema de salud pública global por su carácter epidémico, su elevada morbimortalidad y alto costo, se estima que tiene una prevalencia del $10 \%$ en el mundo y adicionalmente no se puede esperar reducción en los costos del tratamiento, ni una medida preventiva definitiva, por tanto la humanidad se enfrenta a un problema de grandes proporciones y difícil de contener en poco tiempo. En Colombia, según datos de la Cuenta de alto costo, en el 2011 existían 640.492 personas afectadas y de ellas 23.301 en estadio 5 que requieren tratamientos de sustitución renal ${ }^{3}$, dentro de los cuales se encuentra la diálisis peritoneal (DP), que es una técnica que permite a la persona realizar su tratamiento en casa, tener independencia, continuar sus actividades laborales, mantener un mayor bienestar físico y mental y disfrutar de una mejor calidad de vida ${ }^{4}$. Para prolongar las ventajas es necesario el fortalecimiento del autocuidado, que según Carrillo y col. ${ }^{5}$ en las personas en tratamiento sustitutivo se enfoca hacia 6 categorías: el mantenimiento y mejora de la aptitud, mantenimiento y mejora del estado nutricional, recreación como medida de autocuidado, protección y mantenimiento de la fístula arteriovenosa, la terapia farmacológica como medida de autocuidado y desde la experiencia y construcción del conocimiento algunas prácticas de autocuidado.

El autocuidado ha sido fundamentado en Enfermería por Dorothea Orem $(1980)^{6}$, quién lo define como
"Una conducta que existe en situaciones concretas de la vida, dirigida por las personas sobre sí mismas, hacia los demás o el entorno, para regular los factores que afectan su propio desarrollo y funcionamiento en beneficio de su vida, salud o bienestar", concepto importante cuando se refiere a pacientes en DP, porque se realizan ellos mismos el procedimiento y viven su cotidianidad sin supervisión directa del equipo de salud. Teniendo en cuenta la importancia del autocuidado en personas en condición de cronicidad, grupo dentro del cual se encuentran los pacientes en DP, Evers e Isemberg diseñaron la escala ASA-A, cuyo objetivo es medir la operatividad de la capacidad de autocuidado desde la perspectiva del propio paciente $^{7}$.

Enmarcada en este contexto se realizó la presente investigación con el objetivo de describir la operatividad de la capacidad de autocuidado de un grupo de personas que se encuentran en el programa de DP en unidades renales de Bogotá, durante el año 2013.

\section{Material y método}

Diseño de investigación: El trabajo tiene enfoque cuantitativo y utiliza un diseño descriptivo de corte transversal. La población del estudio la constituyen 915 personas con IRC en programa de DP en unidades renales de Bogotá, reportados por la cuenta de enfermedades de alto costo ${ }^{3}$. Se incluyeron los pacientes mayores de 18 años, con IRC diagnosticada por un nefrólogo, con más de 1 mes de permanencia en el programa, que se realizan los recambios a sí mismos, y aceptan voluntariamente participar en el estudio, se excluyeron los pacientes con alteraciones neurológicas, mentales, motoras o cognitivas previas o adquiridas durante el tratamiento, que les impedía para el autocuidado, según la información de los registros clínicos.

Muestra: Se calculó con base en la información de la Cuenta de Enfermedades de alto costo, correspondiente a julio de 2011, que reporta 915 personas en el programa de DP en las unidades renales de Bogotá3, y los resultados del trabajo "Capacidad de autocuidado de los pacientes en diálisis peritoneal: un estudio piloto en Bogotá" el cual estableció que el $60 \%$ de los sujetos presentaba suficiente capacidad de autocuidado ${ }^{8}$.

Con base en lo anterior, se estimó una diferencia máxima del $5 \%$, un error tipo 1 de $5 \%$ y un cálculo a dos colas, dando como resultado una muestra de 261 y se adicionó un $10 \%$ por no respuesta para un total de 
290 pacientes. Una vez aplicada la proporción reportada por la cuenta de alto costo de pacientes en DP manual y automatizada, se obtuvo como resultado 107 (36.9\%) pacientes en automatizada y $183(63.1 \%)$ en manual. Se realizó muestreo aleatorio estratificado proporcional a la cantidad de pacientes en cada una de las unidades renales y al tipo de DP, y se seleccionaron los participantes de cada unidad utilizando el método coordinado negativo.

Estrategias de seguimiento y captación de pacientes: La selección de los pacientes se realizó en coordinación con la enfermera del programa de cada Unidad renal, en la cual fueron abordados el mismo día del control mensual. En el primer contacto se les informó sobre el estudio y se les invitó a un lugar con la privacidad requerida para darles a conocer los objetivos del mismo y el consentimiento informado, el cual firmaron si aceptaban participar, acto seguido se obtuvo la firma del cuidador familiar o un testigo. En caso de respuesta negativa por parte del paciente se asumió dentro del $10 \%$ calculado para pérdida. Una vez diligenciado el consentimiento informado, las enfermeras especialistas en nefrología y urología, previamente entrenadas por la investigadora aplicaron tres instrumentos:

- Cuestionario para identificar características sociodemográficas y variables clínicas.

- Test de Morisky Green.

- Escala Appraisal of Self-care Agency Scale, respondida por el paciente propuesta por Evers e Isemberg en 1983, validada para Holanda en 1993 traducida al español como "Escala de Valoración de Agencia de Autocuidado (ASA-A)", que es un instrumento de 24 ítems, tipo escala de Likert, modificado en Colombia por Edilma de Reales, con autorización de la autora, y validado por Manrique y Velandia, para la población bogotana ${ }^{10}$, la puntuación 1 corresponde a nunca, 2 a casi nunca, 3 a casi siempre y 4 a siempre, por tanto, el sujeto con máxima capacidad de autocuidado tiene una puntuación de 96 y el mínimo de 24. Para la descripción de los resultados reportados por la escala ASA-A se tuvo la precaución de invertir la puntuación correspondiente a los ítems: 6. "Me faltan las fuerzas necesarias para cuidarme como debo", 11. "Pienso en hacer ejercicio y descansar un poco durante el día pero no llego a hacerlo", y 20. "Debido a mis ocupaciones diarias me resulta difícil sacar tiempo para cuidarme", porque por la forma en que están formulados, la respuesta siempre equivale a 1 y nunca a 4 .
Las encuestas fueron transportadas y entregadas por las recolectoras personalmente a la coordinadora del estudio, quien una vez evaluada la calidad de la información con la investigadora principal, comparó los registros con la base de datos enviada por las recolectoras y las archivó conservando la confidencialidad. Para el análisis estadístico se utilizaron frecuencias absolutas y relativas, medidas de tendencia central y de dispersión. La clasificación de la operatividad de la capacidad de agencia de autocuidado sigue lo descrito por Evers ${ }^{1}$ previa equivalencia a los valores de la Escala ASA-A modificada por Edilma de Reales para la población bogotana, por tanto se establecieron los siguientes rangos:

- Alta: puntajes iguales o mayores a 76.

- Media: iguales o mayores a 70 y menores o iguales a 75.

- Baja: 69 o menor.

Mediante un gráfico de perfiles se presentan los porcentajes de respuesta para cada ítem de la escala ASA-A y se realiza análisis de correspondencias múltiples tomando como variable ilustrativa la operatividad de la capacidad de agencia de autocuidado (OCAA) y como variables activas las características sociodemográficas y clínicas de los pacientes. El análisis se realizó con el apoyo de los software Stata $12^{\circledR}$ y Spad $7.3^{\circledR}$

El trabajo cumple los requerimientos éticos establecidos en la Resolución 8430 de 1993 del Ministerio de Salud colombiano ${ }^{11}$, según la cual este estudio se considera sin riesgo y fue aprobado por el Comité de Investigación con Seres Humanos Hospital de San José-Fundación Universitaria de Ciencias de la Salud (CEISH) como consta en el acta $\mathrm{N}^{\circ} 069-2012$. Inmediatamente aplicado el instrumento, la enfermera especialista en nefrología y urología realizó el plan de cuidado de enfermería con base en el déficit identificado, el cuál entregó impreso a cada paciente y en las unidades que lo requirieron, previa autorización del paciente, dejó copia en la historia clínica.

\section{Resultados}

Características sociodemográficas de la población de estudio: La edad promedio de los participantes es 50 años (DE: 15), la persona más joven tiene 18 y la mayor 80; la mayoría de la población es de sexo femenino $(55.2 \%)$, están casados $(37 \%)$, pertenecen al estrato socioeconómico $2^{12}(42 \%)$, cursaron la primaria $(37.2 \%)$ y están desempleados $(25,8 \%)$ como se puede observar en la (tabla 1 ). 
Tabla 1. Características sociodemográficas de la población de estudio.

\begin{tabular}{|c|c|c|}
\hline & n (276) & $(\%)$ \\
\hline Edad, promedio (DE) & 50,0 & (15.0) \\
\hline Mínimo-máximo & 18 & 80 \\
\hline \multicolumn{3}{|l|}{ Sexo } \\
\hline Femenino & 152 & $(54.5)$ \\
\hline Masculino & 127 & $(45.5)$ \\
\hline \multicolumn{3}{|l|}{ Estado civil } \\
\hline Soltero & 73 & (26) \\
\hline Casado & 103 & (37) \\
\hline Viudo & 22 & $(8.0)$ \\
\hline Unión libre & 48 & (17) \\
\hline Separad & 33 & (12) \\
\hline \multicolumn{3}{|l|}{ Nivel Socioeconómico } \\
\hline Estrato 1 & 34 & (12) \\
\hline Estrato 2 & 117 & $(42)$ \\
\hline Estrato 3 & 101 & (36) \\
\hline Estrato 4 & 17 & $(6,1)$ \\
\hline Estrato 5 & 7 & $(2,5)$ \\
\hline Estrato 6 & 3 & $(1,1)$ \\
\hline \multicolumn{3}{|l|}{ Nivel Educativo } \\
\hline Sin estudio & 14 & (5) \\
\hline Primaria & 105 & $(37,6)$ \\
\hline Bachillerato & 88 & $(31,5)$ \\
\hline Técnico & 33 & $(11,8)$ \\
\hline Universitario & 39 & (14) \\
\hline \multicolumn{3}{|l|}{ Ocupación } \\
\hline Empleado & 35 & $(12,5)$ \\
\hline Desempleado & 72 & $(25,8)$ \\
\hline Pensionado & 66 & $(23,7)$ \\
\hline Trabajador independiente & 56 & $(20,1)$ \\
\hline Oficios varios & 50 & (18) \\
\hline
\end{tabular}

Características Clínicas de la población de estudio: La mayoría califica su estado de salud como bueno $(62.4 \%)$, no informan antecedentes familiares relevantes $(38.8 \%)$, la etiología de la IRC más frecuente es la glomerular (45\%), hacen ejercicio $(50.54 \%)$, siguen la dieta indicada (81.4\%) y según el test de Morisky Green, el $61 \%$ no cumple el tratamiento por olvido en la toma del medicamento (tabla 2).
Tabla 2. Características clínicas de la población de estudio.

\begin{tabular}{|c|c|c|}
\hline \multicolumn{3}{|l|}{ Percepción del estado de salud } \\
\hline Malo & 5 & $(1,8)$ \\
\hline Regular & 82 & $(29,4)$ \\
\hline Bueno & 174 & $(62,4)$ \\
\hline Muy bueno & 18 & $(6,5)$ \\
\hline \multicolumn{3}{|l|}{ Antecedentes familiares } \\
\hline Ninguna & 95 & $(38,8)$ \\
\hline Diabetes & 58 & $(23,7)$ \\
\hline Hipertensión arterial & 89 & $(36,3)$ \\
\hline Dislipidemia & 3 & $(1,2)$ \\
\hline \multicolumn{3}{|l|}{ Etiología } \\
\hline Ninguna & 3 & $(1,1)$ \\
\hline Diabética & 39 & (14) \\
\hline Hipertensión arterial & 9 & $(3,2)$ \\
\hline Glomerular & 125 & $(45)$ \\
\hline Vascular & 24 & $(8,6)$ \\
\hline Quística & 10 & $(3,6)$ \\
\hline Otras & 65 & $(23,3)$ \\
\hline \multicolumn{3}{|l|}{ Ejercicio } \\
\hline No realiza & 138 & $(49,46)$ \\
\hline Si realiza & 141 & $(50,54)$ \\
\hline \multicolumn{3}{|l|}{ Dieta adecuada } \\
\hline No sigue & 52 & $(18,6)$ \\
\hline Si sigue & 227 & $(81,4)$ \\
\hline Olvida tomar medicamento & 175 & $(62,7)$ \\
\hline No olvida tomar el medicamento & 104 & $(37,3)$ \\
\hline \multicolumn{3}{|c|}{ Adherencia al tratamiento farmacológico } \\
\hline No es adherente & 175 & $(62.7)$ \\
\hline Si es adherente & 104 & $(37.3)$ \\
\hline
\end{tabular}

Operatividad de la capacidad de autocuidado: EI promedio de OCAA es de 78.7 con (DE) de 16.5; si se considera que las personas que están por encima del promedio tienen suficiente OCAA, el $72 \%$ de los participantes se clasifican en ésta categoría; la mediana se ubica en 78 puntos con rango intercuartílico de 77-88, y la moda es 86 puntos. Tres de los participantes obtienen el máximo de 96 y el de menor puntuación es 27 . En el análisis por ítem la mayoría de los pacientes refieren que nunca ejecutan $(38 \%)$ el número 8 "Cambio la frecuencia con que me baño para mantenerme limpio", la respuesta casi nunca fue asignada por el (31\%) al ítem 11 "pienso en hacer ejercicio y descansar un poco durante el día, pero no llego a hacerlo", como casi siempre el mayor número de sujetos (25\%) responde el ítem 6 "Me faltan 
las fuerzas necesarias para cuidarme como debo, y la respuesta siempre es asignada por el $(84 \%)$ de los sujetos al ítem 4 "Yo puedo hacer lo necesario para mantener limpio el ambiente donde vivo", como se muestra en la figura 1.

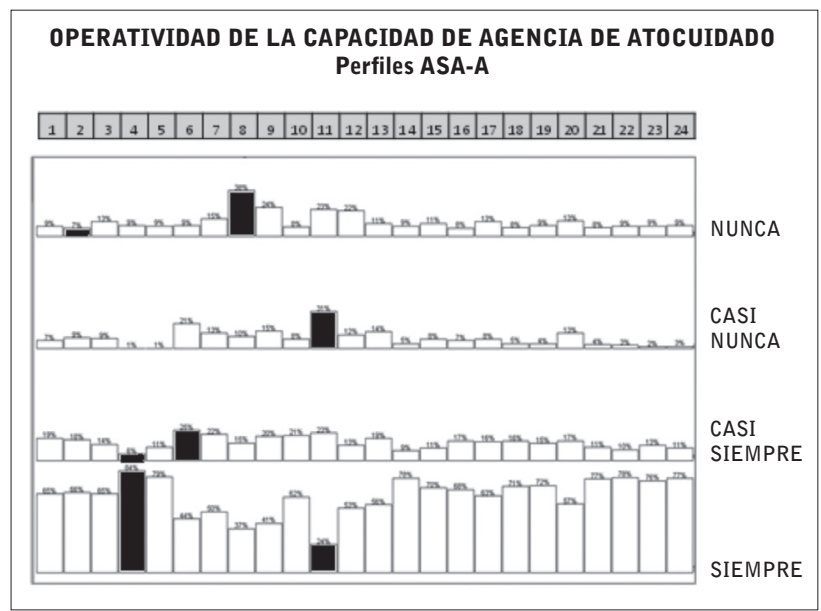

Figura 1. Gráfico de perfiles de los ítems de la escala ASA-A.

Operatividad de la capacidad de agencia de autocuidado según variables sociodemográficas y clínicas: Los resultados reportados por la escala ASA-A con relación a las variables sociodemográficas muestran que las personas clasificadas con OCAA baja se caracterizan por vivir en unión libre, encontrarse desempleados y ser de sexo femenino; los participantes que clasifican en la categoría media se caracterizan por encontrarse entre los 41 a 60 años, estar casados o separados y ser pensionistas, y los que se ubican en la categoría alta son bachilleres y comparten con los clasificados en la categoría baja el vivir en unión libre y encontrarse desempleados, como se observa en la figura 2.

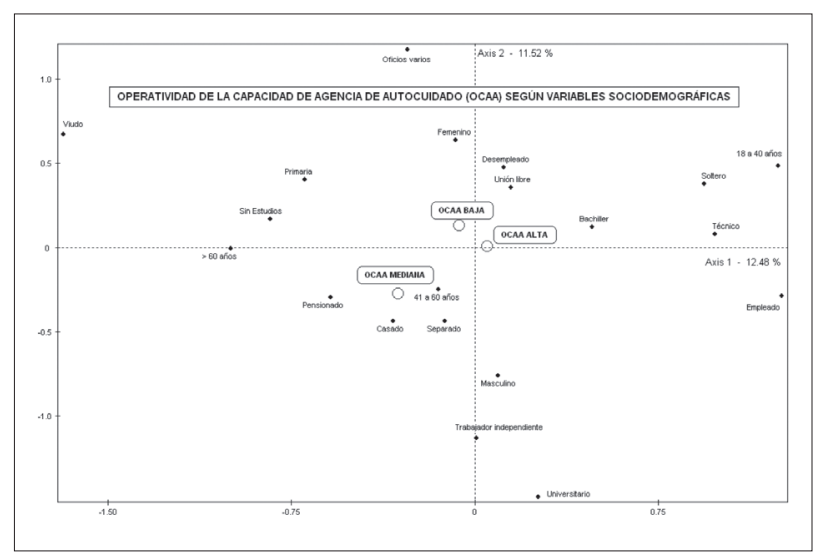

Figura 2. Operatividad de la capacidad de agencia de autocuidado (OCAA) según características sociodemográficas.
La relación de la OCCA y las características clínicas permite observar que los pacientes clasificados en la categoría baja no hacen ejercicio y la etiología de la insuficiencia renal es quística. Los pacientes que se encuentran en la categoría media olvidan tomar la medicación y no siguen la dieta y quienes se clasifican en OCAA alta se caracterizan porque siguen la dieta, no olvidan la medicación, hacen ejercicio y el origen de la IRC es la diabetes mellitus como se muestra en la figura 3.

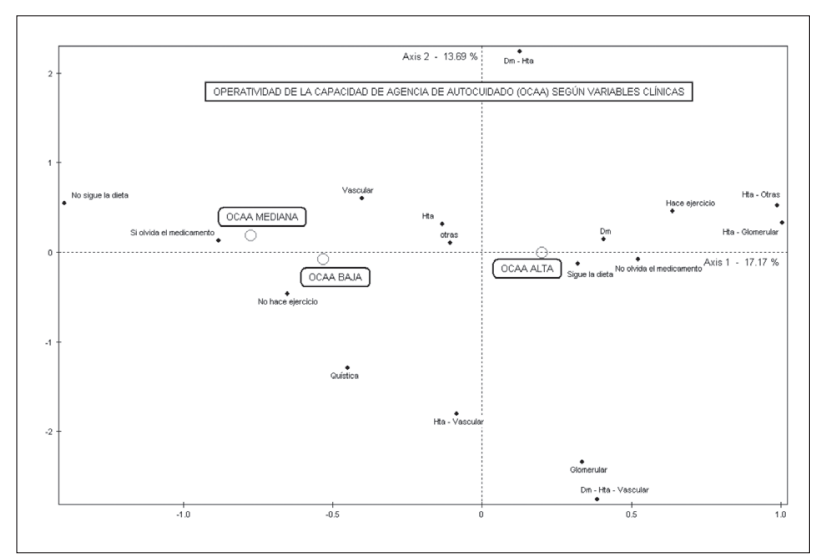

Figura 3. Operatividad de la capacidad de agencia de autocuidado (OCAA) según características clínicas.

\section{Discusión y conclusiones}

La edad promedio es de 50 años con desviación estándar de 15.0, dato inferior a lo reportado en la prueba piloto 8 y acorde con las estadísticas de la cuenta de alto costo que se refiere a la IRC como una enfermedad que afecta a las personas mayores de 50 años, porque como menciona Méndez (2010) ${ }^{13}$ la edad no es un factor determinante para su presencia, pero si es claro que la función renal se deteriora con el paso de los años. En ésta lógica, llama la atención que la persona más joven tiene 18 años, lo cual resalta la necesidad de generar programas enfocados a fortalecer hábitos de vida saludables desde temprana edad. Respecto al sexo, predominó el femenino (55.2\%), resultado acorde con lo reportado por Gámez (2013), ${ }^{14}$ quién constató la prevalencia hospitalaria de IRC en pacientes de 60 años o más, ingresados en la sala de geriatría de un Hospital Universitario y con los resultados del trabajo factores de riesgo para enfermedad renal crónica de Martínez y col (2013) ${ }^{15}$. Desde el punto de vista del estado civil, la mayoría de las personas estaban casadas (37\%) lo cual es esperable teniendo en cuenta la edad promedio de las mismas. Un alto porcentaje (42\%) de los participantes pertenece al estrato socioeconómico dos, hallazgo cohe- 
rente con la prueba piloto ${ }^{8}$ y los resultados del estudio de Martín de Francisco (2009), ${ }^{16}$ quién concluyó que "comparando los dos extremos de nivel socioeconómico, los ubicados en el más bajo presentaban un mayor riesgo de disminución del filtrado glomerular, después de hacer los ajustes a criterios sociodemográficos, de estilo de vida y variables clínicas. El nivel educativo más frecuente es el de primaria $(37.2 \%)$ dato acorde con otros resultados ${ }^{15}$. Laboralmente, el $25,8 \%$ de los participantes está desempleado, aspecto que no concuerda con lo descrito por Julián-Mauro y col. $(2012)^{17}$ quienes encontraron que en España casi la mitad de los pacientes en diálisis peritoneal estaban empleados.

Las características clínicas muestran que a pesar de ser personas en condición de cronicidad, la mayoría considera que su estado de salud es bueno $(62.4 \%)$; un alto porcentaje el $(38.8 \%)$ de los participantes manifiesta no tener antecedentes familiares importantes, respuesta seguida de cerca por la presencia de hipertensión arterial (36.3\%), aspecto a tener en cuenta porque según los resultados de la revisión documental realizada por Ramírez y col. (2011)18 "Se ha estimado que el componente genético es muy importante para su desarrollo, de hecho varios estudios de heredabilidad muestran que entre $30 \%$ a $60 \%$ depende del componente genético para que se desarrolle Hipertensión Arterial (HTA)" y adicionalmente concluyen que ésta enfermedad "es el principal factor de riesgo para el desarrollo de infarto agudo de miocardio, fallo cardiaco, arritmia ventricular, nefropatías (...)". La etiología más frecuente de la IRC fue la glomerular (45\%), hallazgo Ilamativo porque según lo descrito por López (2009)19 "Hoy en día, la disminución en la mortalidad de los pacientes con diabetes mellitus e hipertensión arterial ha permitido que dichas enfermedades evolucionen a complicaciones como la IRC, y han sustituido a las glomérulo nefritis como las causas más frecuentes de enfermedad renal."

El $50.54 \%$ de los participantes hace ejercicio, dato inferior al encontrado en la prueba piloto ${ }^{8}$ que reportó $57 \%$ de las personas, al respecto es importante recordar a Moreno y col. $(2010)^{20}$ en su trabajo con pacientes afectados por IRC, cuando manifiestan que la inactividad física en estos pacientes incluye factores biológicos, sociales o psicológicos. Respecto al seguimiento de la dieta indicada, el $(81.4 \%)$ comenta que la sigue, llama la atención que no se cumpla en el $100 \%$ teniendo en cuenta que Orem $(1996)^{21}$ lo establece como un requisito universal del autocuidado y si se compara con la establecida para personas en hemodiálisis es menos estricta. Según el test de Morisky
Green el $(61 \%)$ de los participantes no son adherentes al tratamiento, se exploraron los cuatro ítems y la mayor causa es el olvido, por tanto la perdida de la memoria es un aspecto relevante en los planes de cuidado y educación dirigidos a pacientes en DP como lo reportan Portolés y col. (2014)22 quienes refieren que el tiempo de entrenamiento en la técnica es más prolongado por problemas de pérdida de memoria.

Respecto a la operatividad de la capacidad de autocuidado, basándonos en los rangos establecidos por Evers $(1989)^{1}$, se clasificaron en categoría alta de OCAA el $77.42 \%$ de los participantes, en la media el $9.3 \%$ y en la baja el $13.98 \%$, es decir que aún hay un $23.3 \%$ de la población con quienes se deben establecer estrategias de cuidado y atención en salud que complementen las actuales con el fin de mejorarla. El promedio de OCAA fue de 78.7 con desviación estándar (DE) de 16.5 , el $72 \%$ de los participantes obtuvo una puntuación superior al mismo superando lo obtenido en la prueba piloto en la cual el porcentaje fue del $60 \% 9$. La mediana se ubicó en 78 puntos con un rango intercuartílico de 77-88, y la moda en 86 puntos, valores que se ubican en la categoría de OCAA alta, lo cual es esperable teniendo en cuenta que tanto el paciente como un cuidador no formal que lo asista tienen entrenamiento cuando ingresan al programa y control mensual en el cual se refuerzan todos los aspectos del autocuidado, sin embargo es necesario analizar cada uno de los ítems de la escala ASA-A, con el fin de identificar en que aspecto especifico es necesario hacer énfasis para que todos los pacientes se acerquen al $100 \%$.

El análisis por ítem mostró que el $38 \%$ de las personas respondió con la opción nunca el ítem "Cambio la frecuencia con que me baño para mantenerme limpio", aspecto a revisar porque los manuales de entrenamiento para el paciente en diálisis peritoneal como el de ALCER Cantabria (2014) ${ }^{23}$ enfatizan que el paciente debe "Mantener una buena higiene corporal como la ducha diaria y el cuidado de las uñas", es necesario recordar que desde la creación de la escala se identificó que éste ítem podía generar diferentes respuestas por las costumbres propias de cada región ${ }^{1}$, sin embargo este resultado, debe llevar al personal de salud a realizar análisis individual para acordar las medidas de higiene necesarias respetando siempre la autonomía de las personas. La respuesta casi nunca fue asignada por el (31\%) al ítem "pienso en hacer ejercicio y descansar un poco durante el día pero no llego a hacerlo" porcentaje similar al obtenido cuando se preguntó directamente si hacían ejercicio, y lleva al personal de enfermería a reforzar en los procesos 
educativos la importancia de la actividad física, teniendo en cuenta las limitaciones para la misma que tienen los pacientes con IRC, descritas en la literatu$\mathrm{ra}^{23}$, en este aspecto se están haciendo esfuerzos con el fin de promover la actividad física, como el de la Universidad Europea Miguel de Cervantes que en su página web publica mensajes como "El ejercicio físico es bueno para todos, incluso para los enfermos de riñón, además de ayudarles con su musculatura, les evita la depresión, la fatiga y mejora su calidad de vida" escrito por García ${ }^{23}$. Es necesario aplicar el sistema de enfermería de educación y apoyo a nivel individual, teniendo en cuenta las diferentes dimensiones del ser humano y la diversidad de contextos, lo cual como lo afirman Mesa y col. ${ }^{24}$ es "todo un desafío para la planificación de abordajes individuales y colectivos pues la dimensión subjetiva y experiencial resultan determinantes en el afrontamiento de los procesos cróni$\cos ^{\prime \prime}$.

Un (25\%) respondió casi siempre al ítem "Me faltan las fuerzas necesarias para cuidarme como debo", al respecto Kamisato y Mauro (2007) ${ }^{25}$ hicieron dinamometría de la mano para medir la fuerza de pacientes en DP comparada con personas normales y encontraron que fue significativamente menor en los primeros demostrando una de las posibles causas de la respuesta de los participantes, e invita al personal de salud a generar programas de acondicionamiento físico que apoyen a las personas en la superación de éste déficit. El $(84 \%)$ de las personas contestó, siempre, a la afirmación "Yo puedo hacer lo necesario para mantener limpio el ambiente donde vivo", aspecto coherente con el resultado de la prueba piloto8 y lo obtenido por Rodríguez $(2013)^{26}$ en pacientes hipertensos, trabajo en el cual, éste ítem logró una de las más altas medias, pero contrario a los resultados del estudio realizado por Rivera $(2006)^{27}$ con pacientes afectados por la misma enfermedad.

Sobre la operatividad de la capacidad de agencia de autocuidado (OCAA) según variables sociodemográficas hemos encontrado que los pacientes ubicados en la categoría baja se caracterizan por ser de sexo femenino, hallazgo que no corresponde con lo descrito por Rodríguez $(2013)^{26}$ en su estudio con pacientes con alteraciones cardiovasculares quien no halló diferencia según género, ni con los hallazgos de los estudios de Velandia (2009) ${ }^{28}$ y Abootalebi col (2012) ${ }^{29}$ en los que las mujeres tuvieron mejor OCAA, pero concuerda con Friedman $(2003)^{30}$ quien encontró que fue mayor en los hombres, por tanto los resultados con respecto al género no son consistentes en los diferentes estudios, y parece no ser determinante en la OCAA; en éste mismo rango se ubicaron las personas sin estudios y quienes cursaron únicamente la primaria, hallazgo comprensible teniendo en cuenta que la operatividad de agencia de autocuidado se incrementa en la medida que las personas desarrollan competencias cognitivas.

Los participantes que clasifican en OCAA media son pensionistas, entre los 41 y 60 años, reporte similar al de Artinian y col (2002) $)^{31}$, quienes encontraron asociación entre el aumento de la edad y mayor probabilidad de realizar algunas conductas de autocuidado, además coherente con Orem (1978) ${ }^{32}$ quién plantea que la capacidad de autocuidado es diferente según la etapa evolutiva del individuo y está determinada directamente por la edad por cuanto depende de la madurez de las personas y de la voluntad para llevar a cabo las acciones. En esta misma categoría se encontró que las personas pueden estar casadas o separadas, lo que parecería indicar que el estado civil no tiene influencia en la operatividad de la capacidad de autocuidado, sin embargo las personas casadas reportaron OCAA media, mientras que los viudos se ubicaron en baja, resultado similar a lo encontrado por Rodríguez (2013) ${ }^{26}$ en cuyo estudio fue mayor en los casados que en los viudos, siendo el estado civil una variable explicativa que se asoció significativamente a tener suficiente capacidad de agencia, este hallazgo es consistente con los resultados de otros estudios reportados por ella $30,33,35$, lo cual invita al personal de salud a revisar cómo fue elaborado el duelo por la pérdida de su compañero/a y como se reestructuró la red de apoyo cuando se produjo la misma.

Los participantes que se ubicaron en la categoría OCAA alta son bachilleres o técnicos, aspecto que concuerda por lo encontrado por Rodríguez ${ }^{26}$ cuando consigna que el nivel educativo se relaciona con el conocimiento que se tenga de la enfermedad, cuanto mayor sea éste, mejor podrá actuar la persona de acuerdo con los cambios de los síntomas de la enfermedad que observa día a día, retomando a Riegel $(2007)^{36}$ y muestran la eficacia de los programas educativos colectivos e individuales para mejorar el cuidado de sí mismo.

Respecto a la operatividad de la capacidad de agencia de autocuidado (OCAA) según variables Clínicas, vemos que los beneficios del ejercicio han sido demostrados en diferentes estudios ${ }^{20,23}$, a pesar de ello la relación de la OCCA con las características clínicas muestran que las personas ubicadas en la categoría baja no hacen ejercicio y el $31 \%$ de los individuos manifiesta que casi nunca lo realiza, al respecto, vale la pena recordar los resultados obtenidos por Junque 
y col. (2013) ${ }^{37}$ quienes demostraron que un programa de entrenamiento físico intradiálisis combinado con electro estimulación mejoró la fuerza muscular y la capacidad funcional de pacientes en hemodiálisis, lo cual invita a reforzar este aspecto en el entrenamiento e iniciar el ejercicio en forma supervisada, teniendo en cuenta que la IRC genera sensación de cansancio y puede existir el temor de desplazar el catéter por el movimiento, por tanto la persona debe conocer los movimientos apropiados y alcanzar la confianza que le permita convertir el ejercicio en parte de su cotidianidad.

Los pacientes ubicados en categoría media olvidan tomar la medicación y no siguen la dieta, aspectos relevantes para el cuidado de enfermería, respecto al cual la "Nursing Diagnosis: definitions and Classification (NANDA)" ha formulado el diagnóstico "Manejo efectivo del régimen terapéutico" el cual se define como "el patrón de integración y regulación en la vida diaria de la persona de un programa para el tratamiento de la enfermedad y de sus secuelas satisfactorio para alcanzar objetivos específicos de salud" y frente al cual invita a analizar aspectos como el déficit de conocimientos o falta de confianza por parte del paciente en el personal de la salud o en el tratamiento mismo ${ }^{38}$, factores modificables con un buen sistema de apoyo educativo. Los participantes clasificados con OCAA alta, se caracterizan porque siguen la dieta, no olvidan la medicación, hacen ejercicio y el origen de la IRC fue la diabetes mellitus y la hipertensión arterial, estas últimas enfermedades generan cambio en el autoconcepto ${ }^{24}$, adicionalmente requieren seguimiento por parte del equipo de salud y la inclusión de quienes las sufren en procesos educativos que mejoran los conocimientos de las personas con respecto a los estilos de vida. En conclusión los resultados de la OCAA ratifican el concepto de 0 rem $^{1}$ cuando expresa que "La operatividad de la agencia en el autocuidado es afectada positivamente por las capacidades de aprendizaje, orientación, comprensión de sí mismo, conciencia de sí mismo, autovaloración y la aceptación, capacidades básicas y disposiciones que están positivamente influenciadas por la subcultura de un individuo en la cual el apoyo social es muy valioso".

\section{Agradecimientos}

El desarrollo del presente estudio fue posible gracias a la Fundación Universitaria de Ciencias de la salud, entidad educativa que aportó los recursos para su ejecución, y a instituciones de salud como el Centro Policlínico del Olaya, Dialy-Ser, ESENSA SAS, Fresenius
Medical Care Colombia S.A, y RTS SAS, entidades que facilitaron el acercamiento a las personas que se encuentran en el programa de DP y cuyos profesionales de la salud aportaron inquietudes que enriquecieron el proyecto.

Recibido: 10 octubre 2014

Revisado: 27 octubre 2014

Modificado: 10 diciembre 2014

Aceptado: 15 diciembre 2014

\section{Bibliografía}

1. Nursing development conference group N.D.C.G concept formalization in nursing: process and product (2nd ed.) Boston: Little Brown and company:1979.

2. Flores $J C$, Alvo $M$, Borja $H$, Morales $J$, Vega J, Zúñiga C, etal . Enfermedad renal crónica: Clasificación, identificación, manejo y complicaciones. Rev. méd. Chile [revista en la Internet]. 2009 Ene [citado 2014 Feb. 23]; 137(1):137-177. Disponible en: http://www. scielo.cl/scielo.php?script=sci_arttext\&pid=S003498872009000100026\&lng=es. http://dx.doi.org/10. 4067/S0034-98872009000100026.

3. Ministerio de Protección social. Cuenta de alto costo pacientes con enfermedad renal crónica en diálisis peritoneal. Bogotá DC, 2011.

4. López-Cervantes M; Rojas-Russell ME; Tirado-Gómez LL; Durán-Arenas L; Pacheco-Domínguez RL; Venado-Estrada AA; et al. Enfermedad renal crónica y su atención mediante tratamiento sustitutivo en México. México, D.F: Facultad de Medicina, Universidad Nacional Autónoma de México. 2009.

5. Carrillo AJ, Laza C, Molina JA. Estudio documental (2006-2013) sobre el autocuidado en el día a día del paciente con enfermedad renal crónica. EnfermNefrol 2013: Julio-Septiembre; 16 (3): 185-192.

6. Marinner A. Raile M. Modelos y Teorías de Enfermería. Elsevier-Mosby. Sexta Edición, 2007.

7. Evers,GCM, Appraisal of self,care Agency A.S.A.Scale: reliability and validity testing of the dutchversiòn of the A.S.A.-scale measuring Orem's concept. Maastricht1989.pág 19-31, 108-122. 
8. Carrillo A.J, Díaz FJ. Capacidad de autocuidado de los pacientes en diálisis peritoneal: un estudio piloto en Bogotá. [consultado el 3 de enero de 2014] disponible en http://revistas.um.es/eglobal/article/view/ eglobal.12.2.163461/147111.

9. Evers. G.C. Eisemberg, M.A. Philipsen.H. Senten M. Brouns. M.N.S Validity Testing of the Dutch translation of the appraisal of the self-care Agency A.S.A. Scale. Int.J.Nurs Stud. 1993;30(4); 331-42.

10. Manrique F, Fernández A, Velandia A. Análisis factorial de la escala de valoración de la agencia de autocuidado. Aquichan,2009(3):203-235.

11. República de Colombia, Ministerio de Salud, Resolución 8430 de 1993.

12. Alcaldía Mayor de Bogotá, Planeación Distrital. La estratificación en Bogotá y estudios relacionado 1983-2004, [consultado 10 de marzo de 2013] disponible en http://www.sdp.gov.co/portal/page/portal/PortalSDP/Informaci\%F3nTomaDecisiones/ Estratificaci\%F3n\%20Socioecon\%F3mica/ QueEs/fee_la_estratificacion_de_bogota_ dc_1983_2004.pdf.

13. Méndez A. Epidemiología de la insuficiencia renal crónica en México. Diálisis y Trasplante 2010;31:711.

14. Gámez AM, Montell $0 A$, Ruano V, Alfonso de León JA, De la Puente M. Enfermedad renal crónica en el adulto mayor. RevMéd Electrón [Internet]. 2013 Jul-Ago [consultado el 10 de enero de 2014]; 35(4). Disponible en: http://www.revmatanzas.sld. cu/revista\%20medica/ano\%202013/vol4\%20 2013/tema01.htm.

15. Martínez ME, Plazas M, Barajas GP, Bravo AM, González C, Rodríguez L. et al Factores de riesgo para enfermedad renal crónica en pacientes que asisten a consulta de medicina interna, [consultado el 10 de enero de 2014]; disponible en; www.actamedicacolombiana.com/ojs/index.php/actamed/ article/download/.../8...

16. Martín de Francisco AL, Piñera C, Gago M, Ruiz J, Robledo C, Arias M, Epidemiología de la enfermedad renal crónica en pacientes no nefrológicos. Nefrología, 2009; 29 (Sup. Ext. 5):101-105.

17. Julian-Mauro JC, Molinuevo -Tobalina JÁ, SánchezGonzález JC, La situación laboral del paciente con enfermedad renal crónica en función del tratamiento sustitutivo renal. Nefrología 2012;32(4):439445 Doi. 10.3265/Nefrologia.pre2012.Apr.11366.
18. Ramírez J. Pérez 0 , Ramírez $S$, Carrillo $S$, Vargas G, Fragoso JM. Genética y genómica de la hipertensión arterial: una actualización, [consultado el 12 de septiembre de 2013] disponible en: http:// zl.elsevier.es/es/revista/archivos-cardiologia-mexico-293/genetica-genomica-hipertension-arterialuna-actualizacion-90028661-articulo-revision -2011 .

19. López M; Rojas ME; Tirado LL; Durán; Pacheco RL; Venado-Estrada AA; et al. Enfermedad renal crónica y su atención mediante tratamiento sustitutivo en México. México, D.F. Facultad de Medicina, Universidad Nacional Autónoma de México. 2009.

20. Moreno $\mathrm{M}^{\mathrm{a}} \mathrm{C}$, Hidalgo M. Á. El ejercicio físico y el paciente renal crónico. Enferm. Nefrol. [Internet] 2012 Dic [citado 2013 junio 23]; 15(4):296-299. Disponible en: http://scielo.isciii. es/scielo.php?script=sci_arttext\&pid=S2254$28842012000400009 \& \operatorname{lng}=$ es.

21. Taylor.S La teoría enfermera del Déficit de autocuidado. En: Marriner A. Raile M. Modelos y teorías en enfermería: Elsevier; 2005: 189-211.

22. Portolés J. Coronel F. Diálisis peritoneal en situaciones especiales. [Internet] [consultado el 14 de enero de 2014] disponible en http://www.valladoliddeporte.es/fasciculo/2012/06/ejercicio-para-losenfermos-de-rinon- 27.

23. García S. Ejercicio para los enfermos de riñón. [consultado el 10 de enero de 2014]; disponible en http://www.valladoliddeporte.es/fasciculo/2012/06/ ejercicio-para-los-enfermos-de-rinon-27.

24. Mesa L, Carrillo AJ, Moreno F. La cronicidad y sus matices: estudio documental, Investigación, enfermería, imagen desarrollo.2013; 15(2):95-114.

25. Kamisato CD, Rivas JJ, Navarro M. Estudio comparativo de calidad de vida, actividad física y fuerza prensil entre pacientes sometidos a Peritoneo diálisis y sujetos sanos, Tesis Entregada a la Universidad de Chile en cumplimiento parcial de los requisitos para optar al grado de licenciado en kinesiología. 2007.

26. Rodríguez $\mathrm{M}^{\mathrm{a}} \mathrm{A}$, Arredondo E. Salamanca YA. Capacidad de agencia de autocuidado y factores relacionados con la agencia en personas con insuficiencia cardíaca de la ciudad de Medellín, [consultado el 20 de diciembre de 2013] disponible en: http://revis tas.um.es/eglobal/article/view/eglobal.12.2.163461 /147111. 
27. Rivera LN. Capacidad de agencia de autocuidado en personas con hipertensión arterial hospitalizadas en una Clínica de Bogotá, Colombia. Rev Salud Pública. 2006;8(3):235-47.

28. Velandia A, Rivera LN. Confiabilidad de la escala "Apreciación de la agencia de autocuidado" (ASA), segunda versión en español, adaptada para población colombiana. Avances Enferm 2009;27(1):3847.

29. Abootalebi G, Vosoghi N, Mohammadnejad E, Namadi M, Akbari M. Study of the self-care agency in patients with heart failure. Iran J Critical Care Nurs. 2012;4(4):203-8.

30. Friedman MM. Gender differences in the health related quality of life of older adults with heart failure. Heart Lung. 2003;32(5):320-7.

31. Artinian NT, Magnan M, Sloan M, Lange MP. Selfcare behaviors among patients with heart failure. Heart Lung. 2002;31(3):161-72.

32. Renpenning K, Taylor S. Self-care theory of nursing: Selected Papers of Dorothea Orem. New York: Springer Pub Co; 2003.

33. Heo S, Moser DK, Lennie TA, Riegel B, Chung ML. Gender differences in and factors related to selfcare behaviors: a cross-sectional, correlational study of patients with heart failure. Int J Nurs Stud. 2008;45(12):1807-15.
34. Lauck S, Johmson J, Ratner P. Self-Care behaviour and factors associated with patient outcomes following same-day discharge percutaneous coronary intervention. Eur J CardiovascNurs 2009;8(3):190-9.

35. Sayers SL, Riegel B, Pawlowski S, Coyne JC, Samaha FF. Social support and self-care of patients with heart failure. Ann BehavMed. 2008;35(1):70-9.

36. Riegel B, Vaughan V, Goldberg LR, Deatrick JA. Factors associated with the development of expertise in heart failure self-care. Nurs Res. 2007;56(4):235-43.

37. Junque A, EsteveV, Iza G, Tomás E, Luceño I, Paz 0, et al. Resultados de un programa de ejercicio físico combinado con electroestimulación neuromuscular en pacientes en hemodiálisis. EnfermNefrol [revista en la Internet]. 2013 Sep [citado 2014 Mar 06]; 16(3):161-167. Disponible en: : http://scielo.isciii. es/scielo.php?script=sci_arttext\&pid=S225428842013000300004\&lng=es. http://dx.doi. org/10.4321/S2254-28842013000300004Gender differences in and factors related to self-care behaviors: a cross-sectional, correlational study of patients with heart failure. Int J Nurs Stud. 2008;45(12):1807-15.

38. Nursing Diagnosis: definitions and Classification, NANDA Internacional, Diagnósticos enfermeros: definiciones y clasificación 2009-2011. 